\title{
Numerical Simulation by CFD of the Growth of Osteocytes within a Bioreactor
}

\author{
Concepción Paz ${ }^{1,2}$, Eduardo Suárez ${ }^{1,2}$, Jesús Vence ${ }^{1}$, Andrea Sande ${ }^{1}$ \\ ${ }^{1}$ School of Industrial Engineering, University of Vigo \\ Lagoas-Marcosende, 36310 Vigo, Spain \\ cpaz@uvigo.es; suarez@uvigo.es \\ ${ }^{2}$ Biofluids Research Group, Galicia Sur Heath Research Institute (IIS Galicia Sur). SERGAS-UVIGO \\ Carretera Clara Campoamor, 36312 Vigo, Spain \\ jvence@uvigo.es; asande@alumnos.uvigo.es
}

\begin{abstract}
For years bone grafts have been used to regenerate or replace damaged bones, treat fractures or bone diseases, which requires surgical intervention and all the risks and problems that this entails, such as: immune response, transmission of diseases etc. As a consequence of the limitations of this technique was born the engineering of bone tissues. The objective of this new type of tissue engineering is to carry out the cellular regeneration of the damaged bone tissue until completely re-establishing its functionality. This requires the creation of artificial porous structures (scaffolding or three-dimensional matrices) biocompatible in which bone cells (osteocytes) extracted from the patient's own tissue are cultured. Achieving adequate oxygen supply, a high cell density and a uniform distribution of cells over three-dimensional scaffolds has become a challenge in recent years. For this reason, a numerical model of osteocyte growth has been implemented in ANSYS Fluent. The model includes the oxygen and nutrient consumption of biomass, the effect of shear stress on cell proliferation and the specific growth rate of osteocytes. This model was evaluated in realistic threedimensional scaffolds inside a perfusion bioreactor and it has been proved that the cell proliferation is conditioned by the wall shear stress.
\end{abstract}

Keywords: Perfusion bioreactor, Scaffold, Osteocytes, Tissue engineering, CFD.

\section{Introduction}

Among the main problems for human health are the loss of organs and the total or partial damage of tissues, caused by external agents, such as accidents, or pathological lesions typical of autoimmune, congenital diseases, cancer, etc. Initially, the solution that arose as a result of this problem and that is still maintained today, was the implantation of prostheses made of materials such as titanium and bone cements. However, these implants can bring with them problems such as postoperative infections, collapse and fracture of the prosthesis or the lack of osseous-integration, that is, of the direct, structural and functional connection between the living bone and the surface of an implant subjected to functional load [1], [2]. Sometimes it is necessary a second surgical intervention, which would significantly reduce the chances of success of the implanted prosthesis. Given these limitations, other alternatives emerged to treat bone defects [3]: One of the most used methodology in recent years is the autografts, it consists of extracting a portion of bone from a healthy area of the patient and transplant it to the affected area of the same. A similar methodology is the allograft, which the bone tissue is transferred between two genetically different individuals but of the same specie (usually the tissue is extracted from a corpse). Finally, the less used technique is the xenograft, in which the tissue transfer is done between individuals of different species.

As a consequence of the limitations presented by the previous techniques, bone tissue engineering emerges. This new discipline aims to create biocompatible and biomimetic artificial structures to carry out the cellular regeneration of damaged bone tissue until the complete restoration of its functionality. For the creation of such osteogenic implants, the combination of three-dimensional scaffolds or matrices and bioactive molecules is necessary [4]. The strategy to follow to obtain this type of implants consists of extracting autologous bone tissue from a healthy area of the patient through a biopsy, from which cells that are introduced into the three-dimensional matrix are subsequently extracted. These cells grow in vitro when subjected to chemical stimuli exerted by growth factors or bioactive molecules, thus forming biocompatible structures that are implanted in the individual. 
Currently, the use of CFD calculations is widely extended for the analysis of biological systems and to study the behaviour of the fluid through the bioreactor [5], [6]. These calculations are carried out to understand the hydrodynamic environment of a bioreactor and the factors that affect it. In addition, the information obtained with CFD also allows to understand the influence exerted by the transport of nutrients on the growth of cells and the effect of cell proliferation on the tissue regeneration [7]. In this way, the effect of mechanical loading on three-dimensional scaffolds can be studied using CFD [8], and this is especially advantageous when designing bioreactors. Modelling with CFD helps to characterize the fluid flow and provides initial estimations. The influence of parameters such as speed, oxygen consumption, shear stress and cell growth through a bioreactor can be studied thanks to CFD simulations [9-11]. The benefits of using these simulations are: integrating the moment and mass transfer equations in a single system, analysing the problems that can occur when scaling the reactor and identifying the best design for the bioreactor, thereby reducing costs and time in the conduct of experiments [12]. The objective of this study is to develop and implement a numerical model of osteocyte growth in CFD, and evaluate this model in a three-dimensional scaffold inside a perfusion bioreactor considering the oxygen and nutrient consumption and the specific speed of growth of the cells.

\section{Physical model}

The cell growth produced in a bioreactor is a complex process, a complete modelling should take into account the cells metabolism, growth and death mechanisms due to other aspects related to age. The rate of oxygen consumption is proportional to the mass transfer, by convection and diffusion basically, and this is translated into an impact on the cell proliferation. Because the cells proliferate and invade the empty space of the three-dimensional scaffold, the porosity and permeability of the scaffold diminish and the space remaining for the appearance of new cells is smaller. This causes the appearance of the phenomenon of contact inhibition, which consists of the cells slowing down their metabolism as their density increases and stops dividing when the critical cell density is reached.

In the present research, a simplified modelling has been carried out, in which differences in the age, shape and size of the cells are not considered, but a detailed description of the oxygen consumption and the growth rate of the cells is taken into account. In addition, the effect of temperature due to the metabolic reaction is disregarded since it can be assumed as a hypothesis that the system is in an incubator in which the temperature is controlled and remain constant. Changes in the density of the culture medium and the $\mathrm{pH}$ resulting from the release of catabolic products are also neglected [13]. A kinetic term is included in the model to consider how cell death affects the rate of growth. However, for an adequate treatment of cell death it would be necessary to take into account the detachment of them. In this study the kinetic term of cell death is considered null, since according to Coletti et al. [13], for the case of osteocytes and the culture time -approximately 7 days - the experiments show that the effects of cell death can be neglected.

Another important effect to be taken into account is that the culture medium that flows through the pores of the threedimensional scaffold exerts a shearing force on the cells. According to Cartmell et al. [14] once the shear stress exceeds 0.05 $\mathrm{mPa}$, cell proliferation and growth is stimulated. On the other hand, if the effort exceeds $56 \mathrm{mPa}$, the cells detach from the wall of the three-dimensional matrix, decreasing the rate of cell growth. Therefore, this restriction of the shear stress has been incorporated in this work so that, when the value of this parameter is not between the maximum and minimum values, there is no cell growth in that zone.

In order to model the osteocyte growth, three essential equations have been used: The oxygen reaction rate, the cell growth rate, and the cell density evolution. The expression that describes the consumption of oxygen, Eq. (1), is of the form of the kinetics of Michaelis-Menten [13]:

$$
R_{O_{2}}=\rho_{\text {cell }} \frac{Q_{m} c_{O_{2}}}{C_{m}+c_{O_{2}}}
$$

Where $R_{\mathrm{O}_{2}}$ is the oxygen reaction rate, $\rho_{\text {cell }}$ is the cells density, $Q_{m}$ represent the maximal oxygen uptake rate, $C_{m}$ is the substrate concentration at which the reaction occurs at half of the maximum rate, and $c_{O 2}$ the oxygen concentration. The 
growth of osteocytes is described through the Contois equation [13], Eq. (2), which takes into account the phenomenon of contact inhibition.

$$
\mu_{\text {cell }}=\frac{\mu_{\text {cell }}^{\max } c_{i}}{K_{c} \rho_{\text {cell }} V_{\text {cell }} \rho_{c}+c_{i}}
$$

The parameters used are: $\mu_{\text {cell }}$ the cell growth rate, $\mu_{\text {cell }}^{\max }$ is the maximum cell growth rate, $K_{c}$ is the Contois parameter, $\rho_{\text {cell }}$ and $V_{\text {cell }}$ are the single cell density and volume respectively, and finally $c_{i}$ is the substrate concentration. The variation of cell density with time is given by the Eq. (3), in which, as stated before, the kinetic parameter of cell death (kd) is considered null. The initial concentration of cells is $1 \cdot 10^{12}$ cells $/ \mathrm{m}^{3}[13]$.

$$
\frac{\partial \rho_{\text {cell }}}{\partial t}=\left(\frac{\mu_{\text {cell }}^{\max } c_{i}}{K_{c} \rho_{\text {cell }} V_{c e l l} \rho_{c}+c_{i}}-k_{d}\right) \rho_{c e l l}
$$

The table 1 shows the values and references of all the parameters required for the Eqs. (1)-(3).

Table 1: Values of the parameters used on the physical model.

\begin{tabular}{c|c|c|c} 
Parameter & Value & Units & Reference \\
\hline$k_{d}$ & 0 & $\mathrm{~s}^{-1}$ & {$[15],[16]$} \\
$Q_{m}$ & $1.86 \cdot 10^{-18}$ & $\mathrm{~mol} /(\mathrm{cells} \cdot \mathrm{s})$ & {$[17]$} \\
$C_{m}$ & 0.006 & $\mathrm{~mol} / \mathrm{m}^{3}$ & {$[18]$} \\
$\mu_{c e x}^{m a x}$ & $1.5281 \cdot 10^{-5}$ & $\mathrm{~s}^{-1}$ & {$[15],[19]$} \\
$K_{c}$ & 0.006 & - & {$[15],[19]$} \\
$\rho_{c}$ & 1020 & $\mathrm{~kg} / \mathrm{m}^{3}$ & {$[15]$} \\
$V_{\text {cell }}$ & $2.5 \cdot 10^{-18}$ & $\mathrm{~m}^{3}$ & {$[19]$}
\end{tabular}

\section{Numerical model}

\subsection{Geometry}

To design the three-dimensional scaffolds, the Voronoi tessellation method [20] has been used. The Voronoi diagrams are fundamental structures within computational geometry since they store in some way all the information regarding the proximity of points. The idea of the Voronoi diagram is fundamentally based on proximity. Given a finite set of points in the plane $P=\left\{p_{1}, \ldots, p_{n}\right\}$ (with $n$ greater than or equal to two) each $p_{j}$ is associated with those points of the plane that are closer or equal to it than any other of the pi with $\mathrm{i}$ other than $\mathrm{j}$. Every point of the plane is thus associated with some pi, forming sets that cover it. There will be points that have the same of two elements of $\mathrm{P}$ and that will form the border of each region.
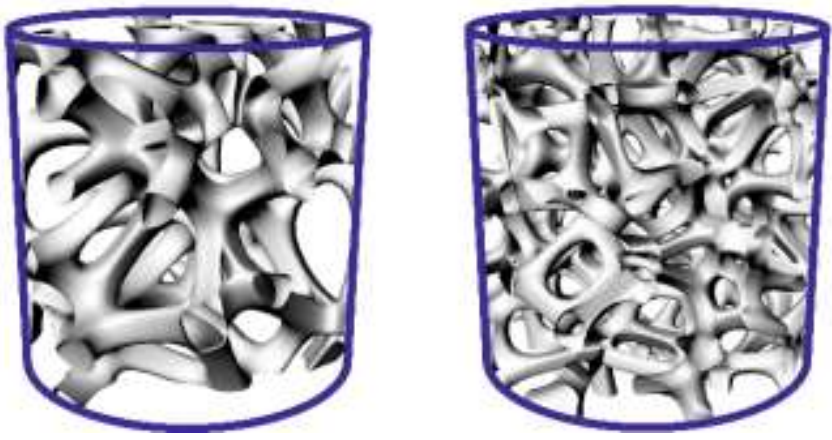

Fig. 1: 3D-scaffolds with a number of initiate set of points: 150 (left) and 500 (right).

The resulting sets form a tiling of the plane, in the sense that they are exhaustive (every point of the plane belongs to some of them) and mutually exclusive except at its border. This tessellation is called a flat Voronoi diagram (denoted by Vor 
$(\mathrm{P}))$. Each of the resulting regions are called Voronoi regions or Voronoi polygons (denoted by Vor $\left(\mathrm{p}_{\mathrm{i}}\right)$ ). The points of the set are called generators in the diagram [20].

The basic principle of the design procedure of three-dimensional scaffolds using the Voronoi diagrams is to define a volume of interest (VOI) whose interior houses the generating points of the diagram. The software Rhinoceros with the graphic algorithm Grasshopher were used for the design of the three-dimensional scaffolds. In this research, a VOI with a cylinder shape of $5 \mathrm{~mm}$ in height by $5 \mathrm{~mm}$ in diameter was used, inside which 150 points and 500 points were introduced to generate the desired scaffolds, Fif.1. The three-dimensional matrix are inside a perfusion bioreactor of $5 \mathrm{~mm}$ in diameter and $20 \mathrm{~mm}$ length.

\subsection{Mesh}

The CFD process always needs the discretization of the fluid domain in control volumes or computational cells. To generate the mesh of the three-dimensional scaffold and the bioreactor, it was used the wrapping tools available on the Fluent Meshing Mode, starting the process with the superficial .stl file created in the previous step. Once a triangular and uniform size superficial mesh was obtained in the scaffold wall, it was filled with tetrahedral cells increasing the size from walls to far region. The mesh of the bioreactor is divided into two fluid cell zones: the three-dimensional scaffold region, and the area of entry and exit of the bioreactor. It was done because the cell proliferation will occur only in the first region; the rest of the bioreactor is simply a passage zone for the fluid. Details of the mesh used are shown on Fig. 2.

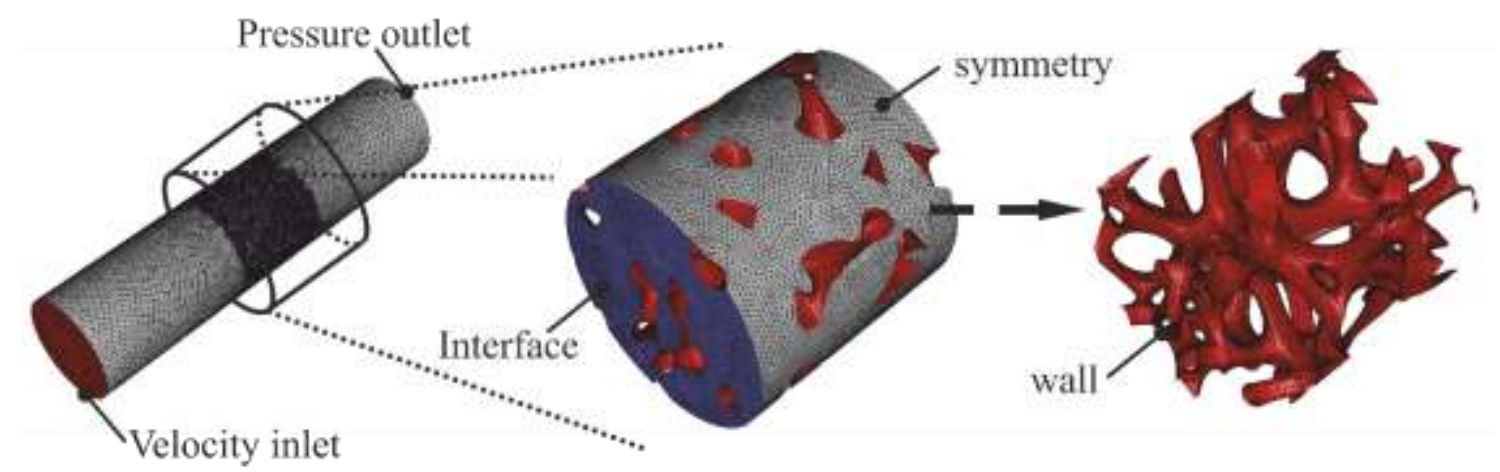

Fig. 2: Details of the mesh used, corresponding to the 150points scaffold, and the different zones.

Due to the computational requirements, associated to the complexity of the growth model implemented, and because it is a three-dimensional and transitory simulation, it was decided to do make meshes as coarse as possible but always taking into account the mesh quality parameters, Table 2.

Table 2: Mesh quality parameters.

\begin{tabular}{c|c|c|c} 
& Mesh elements & Max.Skewness & Orthogonal Quality \\
\hline 150-points 3D scaffold & 293967 cells & 0.955 & 0.037 \\
500-points 3D scaffold & 988082 cells & 0.954 & 0.04
\end{tabular}

\subsection{Set up and boundary conditions}

The CFD software used for solving the fluid flow through the scaffold and the bioreactor was Ansys 15.0 (Fluent Inc.). Because of the laminar behaviour of the substrate flow, the model selected was Laminar model. In addition, it was necessary to use the Species Transport with Volumetric reaction to get into account the oxygen consumption. Cell growth rate and oxygen reaction rate were implemented within the model using User Defined Functions (UDFs).

To simplify the complexity of the calculations, a monophasic model composed of three species has been made. These substances and their estimated physical properties are listed in Table 3. 
Table 3: Species physical properties, and boundary conditions.

\begin{tabular}{|c|c|c|c|c|c|}
\hline \multicolumn{3}{|c|}{ Physical properties } & \multicolumn{3}{|c|}{ Boundary conditions } \\
\hline Specie & $\begin{array}{l}\text { Density } \\
\left(\mathrm{kg} / \mathrm{m}^{3}\right)\end{array}$ & $\begin{array}{c}\text { Viscosity } \\
(P a \cdot S)\end{array}$ & Zone & Parameter & Value \\
\hline $\begin{array}{l}\text { Cells (osteocytes) } \\
\text { Oxygen } \\
\text { Culture medium }\end{array}$ & $\begin{array}{c}1020 \\
1.2999 \\
1009\end{array}$ & $\begin{array}{c}8.4 \cdot 10^{-4} \\
- \\
8.4 \cdot 10^{-4}\end{array}$ & $\begin{array}{l}\text { Velocity } \\
\text { inlet } \\
\text { Pressure } \\
\text { outlet }\end{array}$ & $\begin{array}{l}\text { Velocity }[\mathrm{mm} / \mathrm{s}] \\
\text { Oxygen mass fraction } \\
\text { Cells mas fraction } \\
\text { Oxygen mass fraction } \\
\text { Cells mas fraction }\end{array}$ & $\begin{array}{c}0.02 ; 0.05 ; 0.1 ; 0.2 \\
0.002929456 \\
0 \\
0 \\
0\end{array}$ \\
\hline
\end{tabular}

Oxygen properties were obtained from the Fluent database. The density of a single cell was obtained from the density value of a typical isotonic solution [13], and the properties of the culture medium approximate those of water [21]. The boundary conditions that have been used in osteocyte growth simulations are: velocity inlet, pressure outlet, wall, interface and symmetry, see Fig. (2). The values used for all of them are shown in Table 3.

The concentration of cells that are initially introduced in the bioreactor so that they grow and proliferate is specified through a user defined function (UDF), for that reason in the contour condition of the input a mass fraction of null cells was established. For all the wall type boundaries, the non-slip condition was used, for which ANSYS Fluent uses the properties of the fluid adjacent to the wall to determine the shear stress of the fluid on said surface. For laminar flow this calculation simply depends on the velocity gradient in the wall [22].

First, it is necessary to resolve the flow inside the reactor before implementing the chemical reactions to facilitate the subsequent convergence of the complete model. Once the flow has converged - the residuals have stabilized- all the UDFs are activated and the simulation of the case is continued. For all the simulations carried out in this project, it was used a very small time step size, in the order of $10^{-7} \mathrm{~s}$. For these simulations, the Ansys Fluent Inc. software was used, on an Intel (R) Core(TM) i7-6600U CPU @ 2.60 GHz 2.80 GHz with 16.00 GB.

\section{Results and discussion}

A total of eight simulations were carried out, four velocities of the culture medium in the bioreactor were simulated for each three-dimensional scaffold. In turn, for each scaffold and for each speed cell growth has been simulated taking into account the effect of the shear stress on the wall of the scaffold. The evolution of osteocytes concentration is shown in Fig. 3 , where the concentration at two different moments $-7 \cdot 10^{-6}$ and $2.7 \cdot 10^{-5} \mathrm{~s}-$, is compared. 


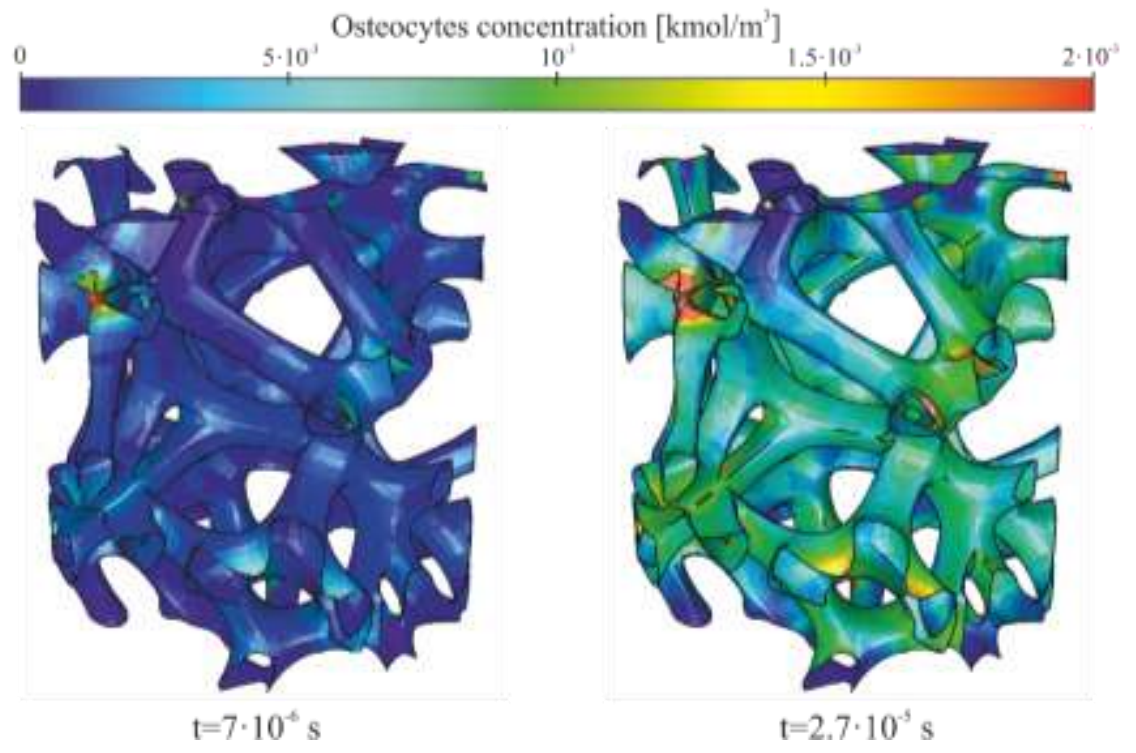

Fig. 3: Evolution of osteocytes concentration $\left(\mathrm{kmol}^{\prime} \mathrm{m}^{3}\right)$ for the 150-point scaffold and $0.02 \mathrm{~mm} / \mathrm{s}$.

At the second moment, when the growth process cover most of the scaffold surface, the concentration contour follow some similarities with the wall shear stress contour.

The influence of the wall shear stress on the growth and proliferation of the osteocytes inside the perfusion bioreactor is evaluated. Fig 4. shows the effect that speed has on cell growth when it is limited by the shear exerted by the culture medium on the wall of the scaffold. In the proposed model, it is considered that there is cell adhesion and cell growth when the shear value is between $5 \cdot 10^{-5}$ and $0.056 \mathrm{~Pa}$, outside this interval the cellular formation process is null. The results obtained shown that the numerical model was implemented correctly because the cellular molar concentration increases with time according to the established by the exponential growth phase of the cells for all the cases simulated. In addition, these figures show that for both types of three-dimensional scaffolds at lower entry speed in the bioreactor of the culture medium, greater growth rate of the osteocytes.

On the other hand, it can be seen that the scaffold of 150 points has a cell growth rate higher than of 500 points with all the simulated speeds. These phenomena are due to shear stress, for the velocity of $0.02 \mathrm{~mm} / \mathrm{s}$ and 150 -point 3D scaffold, there is a greater percentage of wall scaffold areas in which shear forces are produced close to $0.056 \mathrm{~Pa}$, which causes cell growth to be greater for this velocity and scaffold compared to the others. 

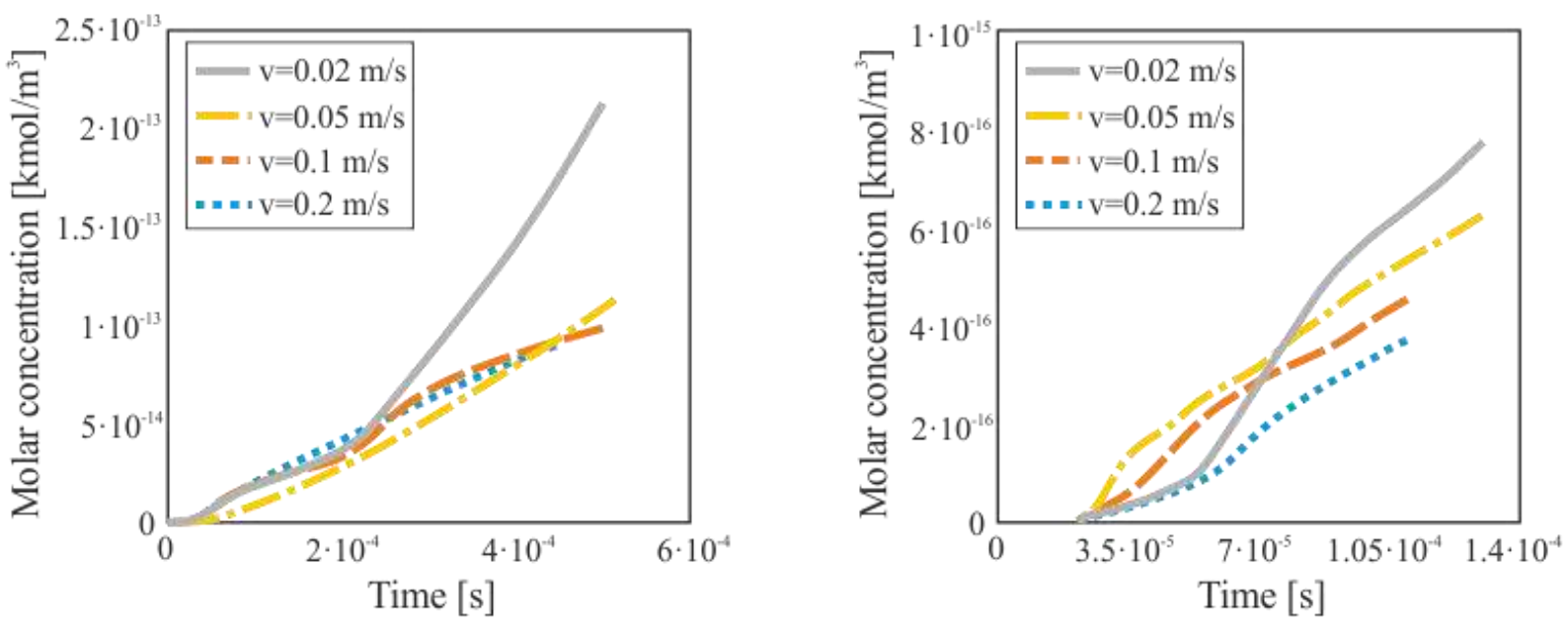

Fig. 4: Evolution of the growth rate of osteocytes at different speeds for the three-dimensional scaffold of 150 points (left) and 500 points (right) when the growth is conditioned by the shear stress.

\section{Conclusions}

A realistic model of osteocytes growth has been implemented in ANSYS Fluent. The cell growth model includes in the same model the oxygen and nutrient consumption of biomass, the effect of shear stress on cell proliferation and the specific growth rate of osteocytes. These parameters have been used separately to describe the growth of osteocytes in previous research, but as far as we known, have not been used integrated in the same model.

The implementation of the model has been evaluated in two three-dimensional realistic scaffolds, of different porosity, and four input speeds of the culture medium in the bioreactor. For all these cases, the results obtained show a cell growth rate with a positive slope, without rises and falls, and sustained over time.

It has been analysed the effect of the different parameters involved in the model, and the cell proliferation is conditioned by the value of the wall shear stress, higher rates of osteocyte growth are obtained for lower entry rates of the culture medium and for three-dimensional scaffolds that are not very porous.

\section{References}

[1] N. Hardt and H. Essig, "Bone Grafts and Specific Implants in Craniofacial Fracture Treatment," in Craniofacial Trauma, Springer International Publishing, 2018, pp. 247-271.

[2] T. Linkevicius, R. Linkevicius, J. Alkimavicius, L. Linkeviciene, P. Andrijauskas, and A. Puisys, "Influence of titanium base, lithium disilicate restoration and vertical soft tissue thickness on bone stability around triangular-shaped implants: A prospective clinical trial," Clinical Oral Implants Research, vol. 29, no. 7, pp. 716-724, May 2018.

[3] B. Zárate-Kalfópulos and A. Reyes-Sánchez, "Injertos óseos en cirugía ortopédica," Cirugía y Cirujanos, vol. 74, no. 3, pp. 217-222, 2006.

[4] M. Cavo and S. Scaglione, "Scaffold microstructure effects on functional and mechanical performance: Integration of theoretical and experimental approaches for bone tissue engineering applications," Materials Science and Engineering: C, vol. 68, pp. 872-879, 2016.

[5] S. Gómez, M. D. Vlad, J. López, and E. Fernández, "Design and properties of 3D scaffolds for bone tissue engineering," Acta Biomaterialia, vol. 42, pp. 341-350, 2016.

[6] G. Mattei and G. Vozzi, "CFD modelling of a mixing chamber for the realisation of functionally graded scaffolds," Computers \& Chemical Engineering, vol. 84, pp. 43-48, 2016.

[7] U. Krühne, D. Wendt, I. Martin, M. V. Juhl, S. Clyens, and N. Theilgaard, "A Transient 3D-CFD Model Incorporating Biological Processes for Use in Tissue Engineering," Micro and Nanosystems, vol. 2, pp. 249-260, 2010. 
[8] F. Zhao, T. J. Vaughan, M. J. M. Garrigle, and L. M. McNamara, "A coupled diffusion-fluid pressure model to predict cell density distribution for cells encapsulated in a porous hydrogel scaffold under mechanical loading," Computers in Biology and Medicine, vol. 89, pp. 181-189, 2017.

[9] I. G. Beskardes, G. Aydin, S. Bektas, A. Cengiz, and M. Gümüsderelioglu, "A systematic study for optimal cell seeding and culture conditions in a perfusion mode bone-tissue bioreactor," Biochemical Engineering Journal, 2018.

[10] H. Kaul, Y. Ventikos, and Z. Cui, "A computational analysis of the impact of mass transport and shear on threedimensional stem cell cultures in perfused micro-bioreactors," Chi. J.l of Ch. Eng., vol. 24, no. 1, pp. 163-174, 2016.

[11] A. Z. Mofrad, S. Mashayekhan, and D. Bastani, "Simulation of the effects of oxygen carriers and scaffold geometry on oxygen distribution and cell growth in a channeled scaffold for engineering myocardium," Mathematical Biosciences, vol. 294, pp. 160-171, 2017.

[12] A. R. Patrachari, J. T. Podichetty, and S. V. Madihally, "Application of computational fluid dynamics in tissue engineering," Journal of Bioscience and Bioengineering, vol. 114, no. 2, pp. 123-132, Aug. 2012.

[13] F. Coletti, S. Macchietto, and N. Elvassore, "Mathematical Modeling of Three-Dimensional Cell Cultures in Perfusion Bioreactors," Industrial \& Engineering Chemistry Research, vol. 45, no. 24, pp. 8158-8169, Nov. 2006.

[14] S. H. Cartmell, B. D. Porter, A. J. García, and R. E. Guldberg, "Effects of Medium Perfusion Rate on Cell-Seeded Three-Dimensional Bone Constructs in Vitro," Tissue Engineering, vol. 9, no. 6, pp. 1197-1203, Dec. 2003.

[15] C. Estrada, A. C. Paz, and L. E. López, "Ingeniería de tejido óseo: consideraciones básicas," Revista EIA, no. 5, pp. 93-100, 2006.

[16] S. Mollazadeh, B. F. Bazzaz, and M. Kerachian, "Role of apoptosis in pathogenesis and treatment of bone-related diseases," Journal of Orthopaedic Surgery and Research, vol. 10, no. 1, p. 15, 2015.

[17] S.-Y. Ho, M.-H. Yu, and C. A. Chung, "Simulation of Cell Growth and Diffusion in Tissue Engineering Scaffolds," in 13th International Conference on Biomedical Engineering, 2009, pp. 1745-1748.

[18] A. G. Mikos, L. V. McIntire, J. M. Anderson, and J. E. Babensee, "Host response to tissue engineered devices," Adv. Drug Deliv. Rev., vol. 33, no. 1-2, pp. 111-139, Aug. 1998.

[19] B. N. Brown, J. E. Valentin, A. M. Stewart-Akers, G. P. McCabe, and S. F. Badylak, "Macrophage phenotype and remodeling outcomes in response to biologic scaffolds with and without a cellular component," Biomaterials, vol. 30, no. 8, pp. 1482-1491, Mar. 2009.

[20] S. Gómez, M. D. Vlad, J. López, and E. Fernández, "Design and properties of 3D scaffolds for bone tissue engineering," Acta Biomaterialia, vol. 42, pp. 341-350, Sep. 2016.

[21] Z. Zhang, L. Yuan, P. D. Lee, E. Jones, and J. R. Jones, "Modeling of time dependent localized flow shear stress and its impact on cellular growth within additive manufactured titanium implants," Journal of Biomedical Materials Research Part B: Applied Biomaterials, vol. 102, no. 8, pp. 1689-1699, Mar. 2014.

[22] ANSYS, ANSYS fluent theory guide. Canonsburg: ANSYS Inc., 2015. 